\title{
Anti-Aging by longitudinal magnetic waves: A new approach by modulating ATP-Levels
}

\author{
Heide Schnabl, Hermann Schnabl, Konstantin Meyl* \\ First Transfer Center of Scalar wave Technology (1st TZS) Erikaweg 32 D-78048 VS-Villingen, Germany
}

\begin{abstract}
Mainstream medicine usually uses drugs as remedy that are expected to interact biochemically with the body's cells to achieve its healing goals. For this purpose, the drug is usually applied orally or topically, as it must come into close contact with the target cells in the body to act according to the biochemical paradigm.

In this context, the question arises, whether longitudinal electromagnetic waves (SW), which have been described as carriers of energy and information in several publications [1,2], are able to cause energy increase (measured by ATP-increase) in the mitochondria of a plant. Since mitochondria exist in the cells of plants as well as in those of animals and humans, and their product - ATP - is the same and indis-pensable for all life processes, the results of this research could also be important for medicine. Moreover, it should be elucidated whether the information of a drug could be transferred using SW [3-6].
\end{abstract}

\section{Introduction}

There are three types of electromagnetic waves, the first, discovered in 1888 by Heinrich Hertz (= the Hertz' wave, transversal) which can be described by the Maxwell equations, the second, an electrical longitudinal wave or scalar wave (SW), discovered and demonstrated by Tesla around 1890 (= Tesla wave, longitudinal), and third, the so called magnetic SW (discovered by K. Meyl in the 1990ies) which plays a crucial role in biology $[1,2]$. However, until now the scalar waves are mostly denied by physicists because the Maxwell equations in today's notation cannot explain SW without additional assumptions. It is important to know that the third Maxwell equation (with div $\mathrm{B}=0$ ) was implicitly extended by the Helmholtz Society in 2009 (resulting in div B> 0, a scalar) by the discovery of Magnetic Monopoles. Unintendely, this discovery results in scalar waves.

While the Hertzian waves have a transversal wave characteristics, whose propagation front is spherically from the sender with fundamentally quadratic decreasing of energy density, both scalar waves are longitudinal waves, which are based on a direct trajectory from the sender to the receiver. For the Tesla wave it is identical with the electric field vector, while for the magnetic SW the trajectory is identical with the magnetic vector. The emitted energy, as was already proven by Tesla, is completely collected again by the receiver. This however, applies only if the state of resonance between transmitter and receiver is accomplished, which requires the same waveform, the same frequency and the opposite phase, conditions normally given with technical devices. It is clear that this condition is also a prerequisite for a full energy-transfer, which cannot be represented by the Hertzian waves because of their quadratic energy decrease. In Figure 1 the characteristics of the 3 wave types are compared.

While technical applications typically use the electrical scalar wave (Tesla Wave), in a biological context, the so-called magnetic scalar wave, discovered by Meyl [3-5] dominates. The theoretical potential of magnetic scalar waves could also better explain why it is possible, that every second in every cell up to 10,000 different biochemical transactions take place in the intra-cell communication process. The mainstream transaction theory of chemistry, following the basics of thermodynamic laws seems not to be sufficient to explain this phenomenon.

\section{Materials and methods}

As a generator for scalar waves in our experiments a recent development of Meyl's experimental set, the so-called "cell radio" was used at a power of $10 \mathrm{~mW}$ for $90 \mathrm{sec}$ (Figure 2, left picture). As a biological substrate we used the flowers (blossoms) of the plant Ipomea (Ipomea purpurea). The flowers were attached to the 'handle' of the cell radio (at a length of their stems of about $10 \mathrm{~cm}$ ) with the help of wet paper pulp, so they could well respond to the frequency pulses. A one-time transmission of a SW-pulse $(90 \mathrm{sec})$ with about $6.7 \mathrm{MHz}$ at the transmitter power already mentioned of $10 \mathrm{~mW}$ to the closed bud provided the plant with the necessary SW-pulse.

Two series of experiments were carried out: In series 1 of the experiments, the described SW pulse without any modulated information was used for the Ipomea purpurea - selected as a model plant for aging because of its relatively precise senescence process whithin $24 \mathrm{~h}$. As parameters for the aging process the blossom diameter (in $\mathrm{cm}$ ) and the ATP content of the individual blossoms in the different stages (Figure 3), based on the weight of the blossom (in g, without stems and sepals) were taken.

In series 2 of the experiments, the SW-pulse was modulated using as information source various biochemicals (e.g. ATP, ubiquinol,

${ }^{\star}$ Correspondence to: Konstantin Meyl, First Transfer Center of Scalar wave Technology (1st TZS) Erikaweg 32 D-78048 VS-Villingen, Germany, E-mail: meyl@k-meyl.de

Key words: aging, longitudinal magnetic waves, ATP-levels, ipomea purpurea

Received: August 23, 2019; Accepted: September 03, 2019; Published: September 06, 2019 


\begin{tabular}{|c|c|}
\hline \multicolumn{2}{|c|}{ Properties of the wave types } \\
\hline $\begin{array}{l}\text { * Transverse wave } \\
\text { * Electromagnetic } \\
\text { Wave (Hertz) } \\
\text { e.g. Radio wave }\end{array}$ & $\begin{array}{l}\text { * longitudinal wave } \\
\text { * electrical or I magnetic } \\
\text { scalar wave } \\
\text { e.g. for the transmission of } \\
\text { energy (Tes/a) information(DNA,Meyl) }\end{array}$ \\
\hline * Energy dissipation & * Energy conservation \\
\hline $\begin{array}{l}\text { * Propagation: } \\
\text { Spherical with } \\
v=c(=\text { constant })\end{array}$ & $\begin{array}{l}\text { * towards the } \\
\text { electric I magnetic } \\
\text { Field pointer (with } v=\text { arbitrary) }\end{array}$ \\
\hline
\end{tabular}

Figure 1. Comparison of the properties of the three types of waves

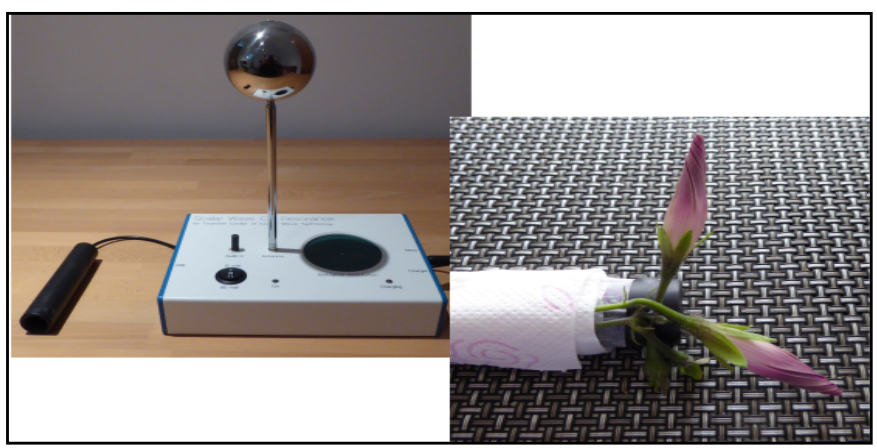

Figure 2. The experimental setup with the 'cell radio' (left), handle with flowers (right) in the bud stage

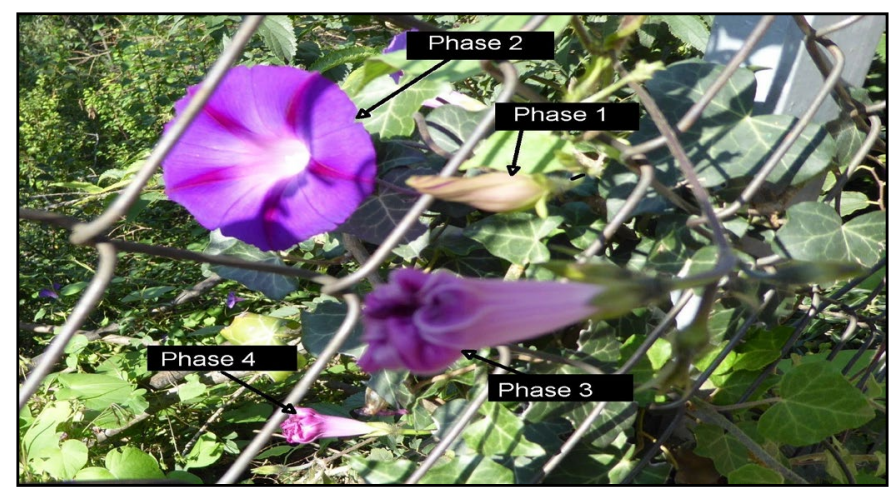

Figure 3. Four typical flowering stages of Ipomea purpurea: Phase $1=$ bud, phase $2=$ opened stage of the blossom, phase 3 = begin of wilting, phase 4 = wilted stage

fullerenes) and various nutrients (resveratrol, curcumin, pomegranate, blueberries, olive, oliphenolia, olive leaf extract, red wine, radish, onions, garlic juice). They were positioned in a liquid form upon the coil in the middle of the experimental set (Figure 2, left picture, the circular part right of the antenna). The blossoms of the Ipomea used were in the bud-stages.

After determining the weight, each blossom was individually macerised by a mixer $(18,000 \mathrm{rpm}, 2 \mathrm{~min})$ in $300 \mathrm{ml}$ distilled water. Subsequently, an aliquot of $100 \mu \mathrm{l}$ was taken in order to measure the ATP content in a luciferin/luciferase system (Flexion.doccheck.com/ luciferin-luciferase). The result is given in so-called RLUs (=Relative Light Units on a linear scale).

\section{Results}

\section{Using SW-treatment without information}

Flourishing process with/without SW: Figure 4 shows that the different stages of flowering are correlated with a change in the diameter of the blossom (in $\mathrm{cm}$; Figure 4, blue columns = control, red columns $=\mathrm{SW}$ ). The application of the SW pulse resulted in an increase of the diameter by about $20 \%$ compared to the control (from 4.8 to 5.8 $\mathrm{cm}$, at $7 \mathrm{am}$ and $9 \mathrm{am}$ ). Moreover, we measured an extension of the senescence period by $2 \mathrm{~h}$, since this reduction of diameter of blossoms as an indicator of aging begins at $11 \mathrm{am}$ in the controls (blue) but at 1 $\mathrm{pm}$ in SW-treated blossoms (red). This is a difference of $2 \mathrm{~h}$, almost $10 \%$, with respect to the total lifespan of the blossoms.

Modulation of ATP: As we see in Figure 5, due to a single SWpulse, the ATP-content of the bud was initially raised by about $40 \%$ against the controls, which however decreases afterwards to a final ATP-content at the wilted stage, which is identical then with that of untreated plants. This initial ATP-difference of $40 \%$ is consistently 'consumed' along the opening of the SW treated buds. The wilting process of SW-treated buds is prolonged by about 2 hours in contrast to controls (Figures 4 and 6). Thus, the initial excess-energy (Figure 5 ) is consumed for an extended flowering process and a lifespan extension by about $10 \%$.

Change of colour: Another finding is the difference in colour comparing SW-treated flowers with the controls at the same stage. Both Ipomea plants (treated/untreated), harvested from the same shrub showed the same red colour at the beginning of the experiment (at 9 am (Figure 4), but only the SW-treated blossoms changed their colour from red to deep violet (Figure 6, right).

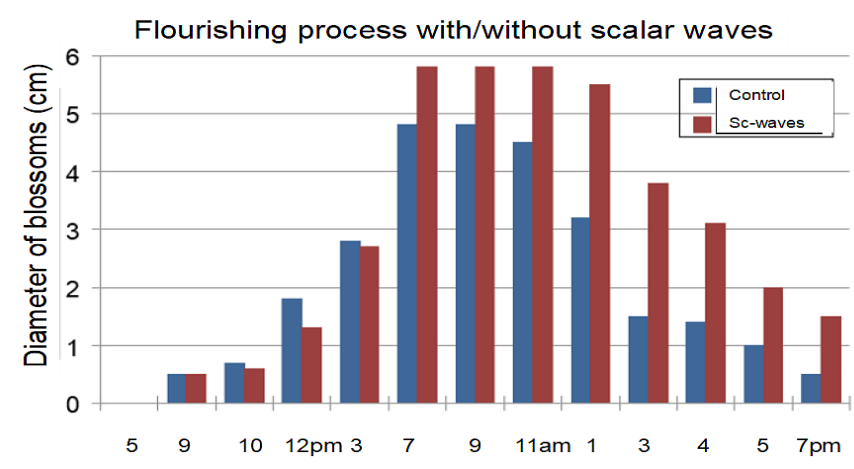

Figure 4. The $24 \mathrm{~h}$ aging-process of the flowers, measured in $\mathrm{cm}$ of the diameters (controls blue, SW-treated, red)

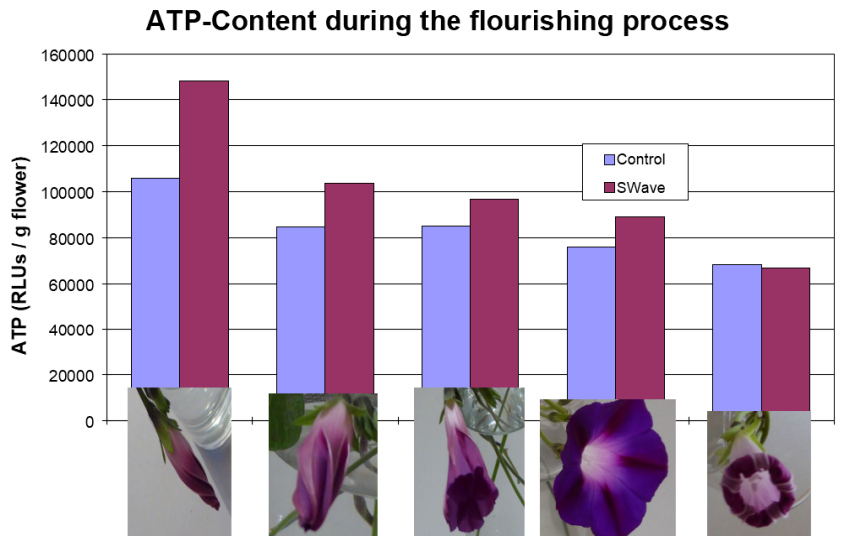

Figure 5. The ATP-level in the buds increased by $40 \%$ due to SW-treatment $(10 \mathrm{~mW}, 90$ sec, red columns in contrast to controls, blue).This ATP difference is metabolized during the flowering process thus delivering energy for a prolongation of the flourishing process by ca. $2 \mathrm{~h}$ ( $\sim 10 \%$ of the life span) 
Since the Ipomea purpurea blossoms contain antocyanidines which are known to change colour like shown in Figure 7 [6], due to a change of the $\mathrm{pH}$-level, a change from a phenolic to a chinoidal stage seems also to have happened, triggered by the SW-treatment.

\section{Using SW with additional information}

While the first series of experiments only used the pure SWenergy, in the $2^{\text {nd }}$ series we applied 'energy + information' (means informed resp. modulated SW). The information source (various liquid polyphenolics) was located upon the coil of the transmitter set as described in 'Material and Methods'.

Figures 8-10 show the ATP-increase (between 15 to 82\%), triggered by SW-pulse, which was 'informed' by different polyphenolic sources in contrast to samples with SW alone.

Figure 8 shows the effects of ATP-solutions, acting as information sources, diluted by a factor of $100(\mathrm{D} 9, \sim 0.5 \mu \mathrm{Mol}$ ATP ), 1000 (D12) and again 1000 (D15) compared with ATP-D7. This results show that information of a homeopathic ATP solution increased the ATP-levels between $15 \%$ (ATP-D7) to $68 \%$ (ATP-D9) again, in comparison to the ATP-level given by an SW-treatment alone.

Figure 9 displays the results of the 'energy + information' experiments using the following nutrients as information sources:

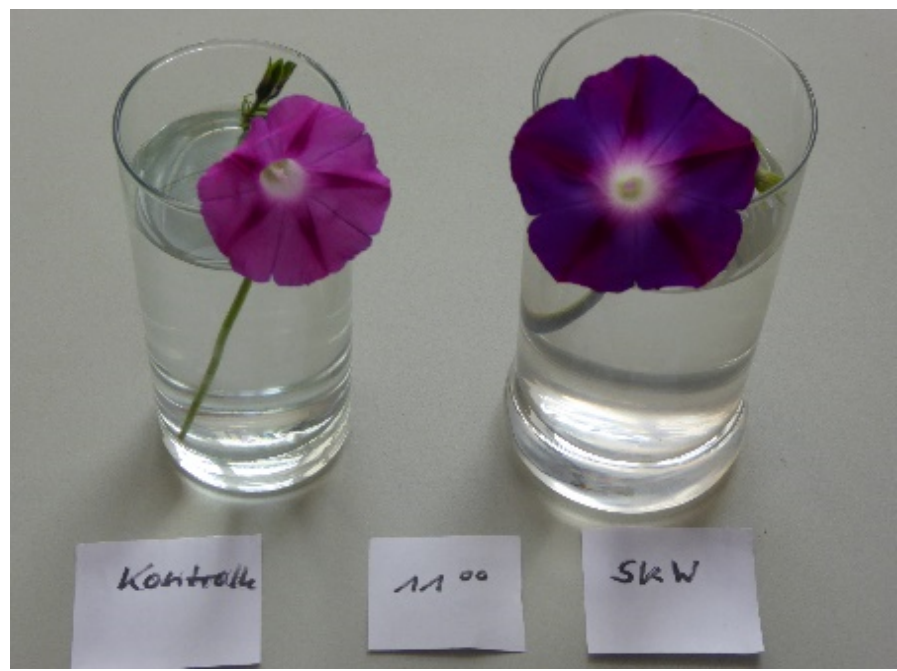

Figure 6. The SW-treated flower (right) shows a deep violet colour in contrast to the control, (left, red), which reflects a change from a phenolic to a chinoidal stage of antocyanidines

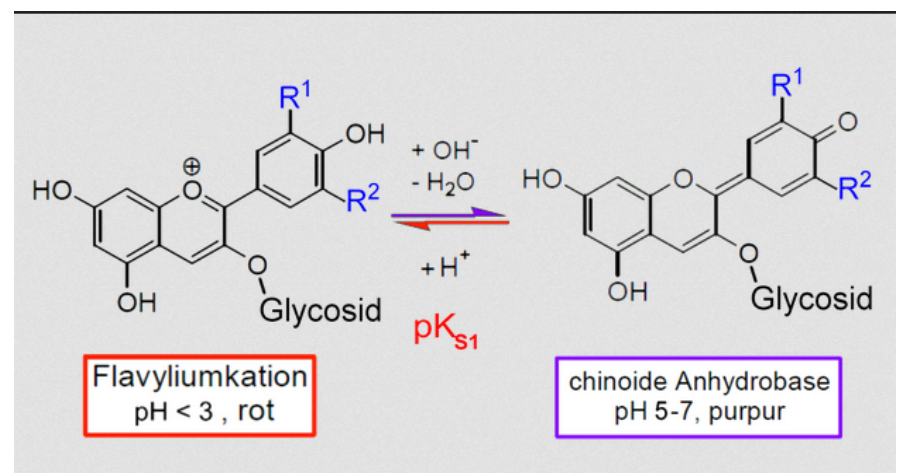

Figure 7. Redox state of anthocyanins: colour changes as a function of the phenolic resp. the quinoide state of the anthocyanins (see left: red, phenolic state, purple, quinoidal state). The colour change is depending on $\mathrm{pH}$ (Source: [6])

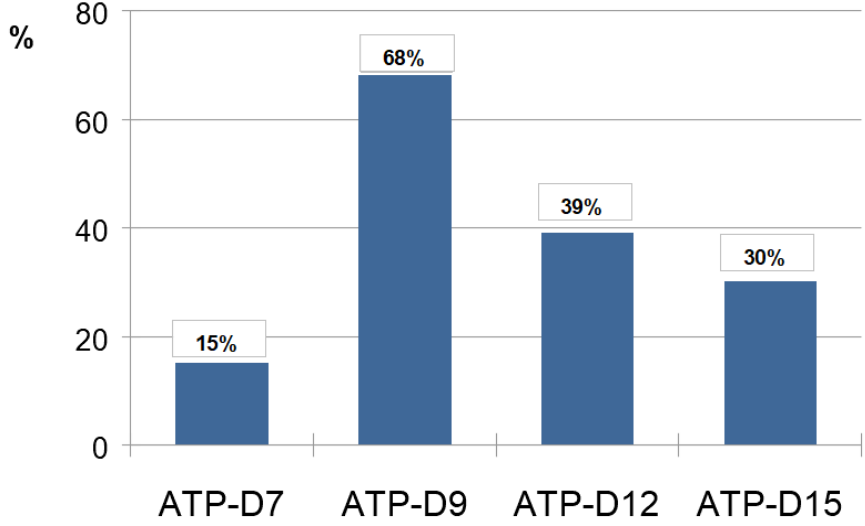

Figure 8. Homeopathic ATP-levels (D7-D15) used as information sources, trigger additional ATP-contents (in \%)

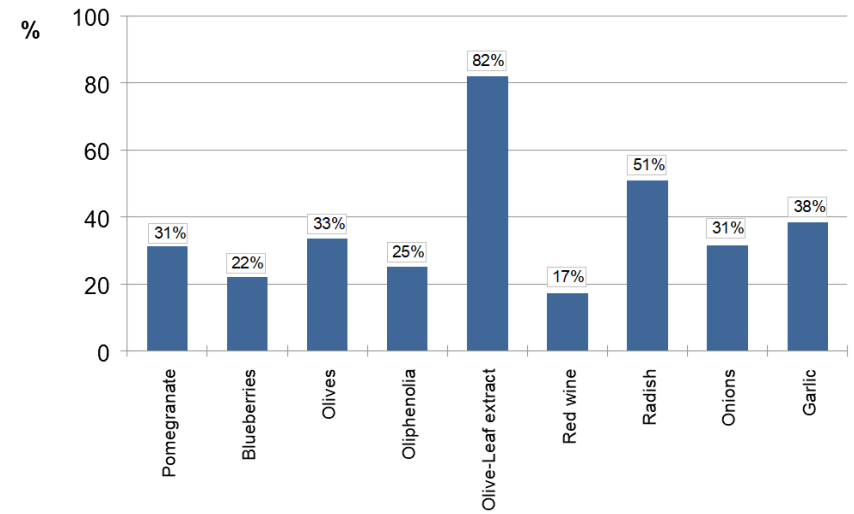

Figure 9. Various nutrients as information sources trigger additional ATP-contents (in \%)

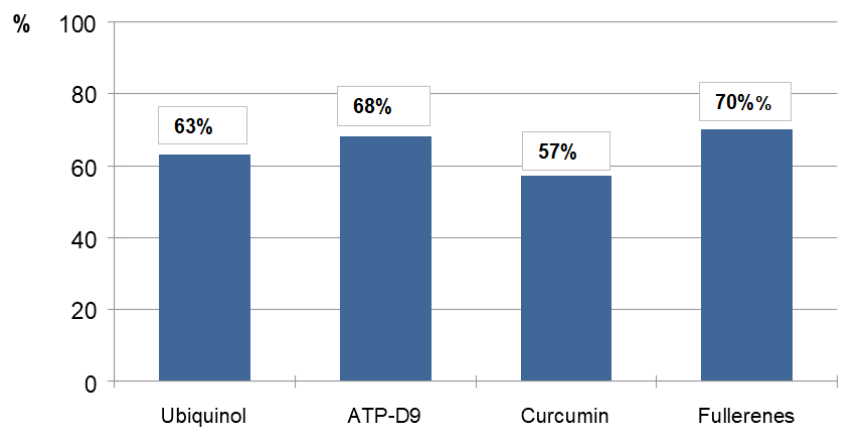

Figure 10. Various biochemicals as information sources trigger additional ATP-contents (in \%)

pomegranate seed extract $(+31 \%)$, blueberries $(+22 \%)$, olives $(+33 \%)$, oliphenolia $(+25 \%$, a liquid water extract of the olive extraction process), olive leaf extract $(+82 \%)$, red wine $(+17 \%)$, radish extract $(+51 \%)$, onion extract $(+31 \%)$ and fresh garlic extract $(+38 \%)$.

In Figure 10 the additional ATP-increase is demonstrated which is due to the additional 'information' of ubiquinol (+63\%), ATP-D9 again as a 'standard', curcumin $(+57 \%)$ and fullerenes $(+70 \%$, a fullerene is as molecule similiar to a hollow sphere, like a football, consisting of a mesh of regular hexagonal rings). 


\section{Discussion}

Since ATP as the 'energy-currency' of plants, animals and humans is produced in the mitochondria as a result of a functioning respiratory chain, the intervention of the longitudinal waves is hypothesized to take place there. In some earlier papers it was proposed, that mitochondria, located inside tunneling nano tubes are able to form a connected structure in order to exchange energy and signals (information) between cells electrically or electro-magnetically $[7,8]$.

Along the respiratory chain in the mitochondria reduction equivalents of NADH via co-factors reduce the oxygen from the air to water. To synthesize ATP, four defined enzyme complexes transport electrons through the inner mitochondrial membrane, which at the end of the chain reduce oxygen to water. The simultaneously released protons are pumped into the intermembrane space. The resulting proton gradient is the prerequisite for the formation of ATP via the ATP synthase complex. There is a necessity for the presence of a number of co-factors for the respiratory chain in order to function, e.g. CoQ10 (ubiquinol) plays a key role as an electron donor. Without ubiquinol, ATP is not sufficiently provided to the cells [9]. If the respiratory chain does not work sufficiently along the 4 complexes, resp. a single mechanism is blocked as a result of diseases, aging processes or drugs, environmental toxins, electro smog, nitrosative stress, the reduction to water may be interrupted and intermediates like the reactive oxygen radicals (ROS) are built up as the source of oxidative stress. Thus, the functioning of mitochondria including the respiratory chain is a fundamental basis of every living organism.

On this basis there arise 4 questions:

1. How does the SW-pulse translate the energy into biochemical reactions, especially into the higher ATP-level?

2. Why does the increase in ATP prolong the lifespan of Ipomea blossoms?

3. 3- How can the structural information of a chemical or of a nutrient be transferred to a biological recipient (such as a plant), using SW?

4. What do the results mean for our medicine?

\section{Question 1}

Like broadcasting a symphony by radiowaves -- which means modulating the transversal waves with the acoustic information -- it is also possible to modulate longitudinal waves with certain organic or chemical 'informations' using SW as carrier waves. As it was shown already in $[1,2]$, magnetic SW are able to transfer information produced by a cell itself. In the range of cell to cell signalling, a magnetic SW-pulse is used by the cell in order to communicate intra- and intercellularly. The effect of a SW-pulse on biochemical structures seem to work via delocalized electrons in their ring-structures. A special example of this is the phenomenon of mesomerism (Figure 11).

This process is offered in more detail: A SW-pulse induces a rotation of the delocalized electrons. This results in the following additional options:

The rotation acts as

- an antenna,

-storage element

- an energy source again, applied for other cellular processes (e.g. in mitochondria)
On this basis of the response to a SW pulse, a phenolic ring can act as an antenna, as a buffer or as an energy source for further pulses. Thus biochemical processes are assumed to be selectively triggered due to resonance conditions because of the similarity of the participating biochemical structures. In this way one can imagine that an ATP signal causes more ATP in the mitochondria.

A further process, which is shown in Figure 12, can also contribute to an increased ATP-production: An excess of rotational energy can also cause electrons to loose the mesomeric attachment, as shown by the red arrow in Figure 12. In this case, the number of free electrons increases contributing to a higher ATP-amount in the mitochondria. Thus, the release of electrons is stimulating the efficiency of mitochondria and antioxidative effects simultaneously.

The observed colour change of the blossoms (from red to a deep violet) reflects the transition from a phenolic to the quinoidal state of antocyanins, contained in the red/purple blossoms of the Ipomea (see Figures 6 and 7), resulting in the release of electrons.

\section{Question 2}

The question of why an ATP increase extends the lifespan of the flower by almost $10 \%$ could be explained by the increased availability of the 'energy currency' ATP. Since ATP is necessary for all biochemical processes, it should be hypothesized that an external SW-pulse translated into more ATP also produces more protective enzymes within the mitochondria. Ultimately, this relative abundance of ATP also supports a prolonged protection of the mitochondria themselves, which also results in an extension of the lifespan of flowers.

\section{Question 3}

The $3^{\text {rd }}$ question about the kind of a transport-mechanism for information via an SW-pulse is innovative in this area.

The SW-pulse was hypothesized to be absorbed via mesomery, typical for organic ring molecules of plant polyphenols, such as the

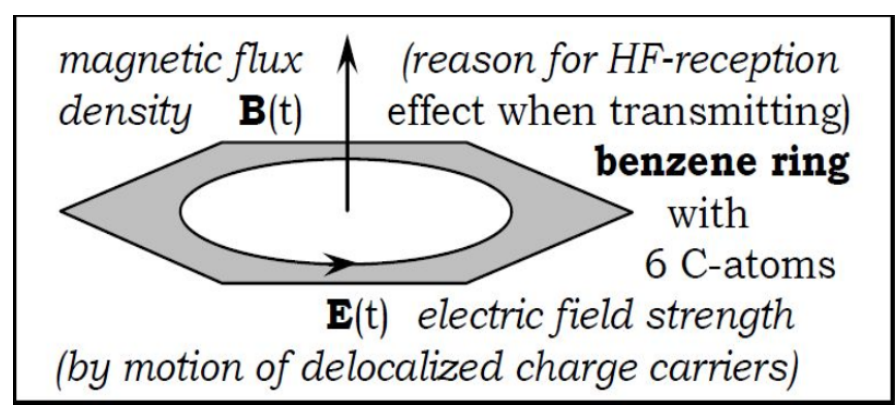

Figure 11. Connection between electromagnetic field impulse and biochemistry

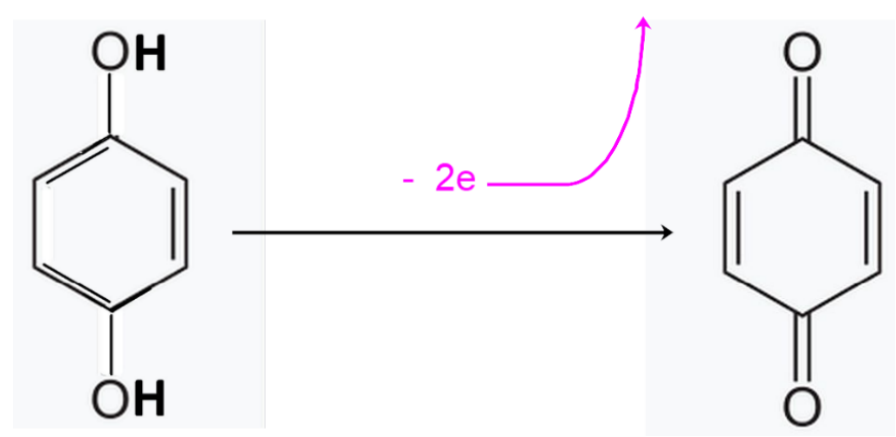

Figure 12. Transition from the phenolic to the chinoidal stage within plant polyphenolic molecules such as the anthocyanins 
anthocyanins. Polyphenols - like resveratrol (e.g. in red wine), quercetin (e.g. in onions) or CoQ10 - are assumed to function as 'antenna molecules' absorbing, storing and releasing the energy of SW pulses. Subsequently, these energy pulses get modulated if they are taking up specific information incorporated in polyphenolics. They also seem to be transferred via co-factors, within the respiratory chain, e.g. CoQ10, thus stimulating the electron flow. This phenomenon is correlated with an increased ATP level optimizing the functionality of the mitochondria and the biochemical processes concerning polyphenolics.

As shown in Figure 8, the degree of dilution of liquid ATP plays a key role in producing ATP. In contrast to the D9-ATP dilution (+68\% ATP) the other dilutions (D7, D12, D15) produced only between $15 \%$ to $39 \%$ ATP. Obviously, the relative degree of freedom of molecules in the dilution is a kind of prerequisite for the quality of signalling.

As shown in Figures 9 and 10, phenolic structures have the potential to increase ATP production. The substances in Figure 9 are nutrients consisting of mixtures of polyphenols, which result in an ATP-increase induced by the degree of polyphenol-mixtures as well as their dilution.

From Figure 10 it is concluded that there exists a positive correlation between the number of phenolic or benzene and pentose rings in the information source and the increase of the ATP level in the biological recipient. However this correlation - as an effect of mesomerism seems to be weakened additionally by the electromagnetic specifities of adjacent rings. Further research in this area is necessary [10].

\section{Question 4}

In summary, sending biochemical information to a biological recipient using an SW-pulse could be a rather innovative enhancement of medical applicability. Information transmitted by SW reaches all cells of the biological recipient and it seems to achieve this effect faster. This is explained by the time-consuming necessity for the drug to permeate the membranes of the recipient, whereas the information via SW-pulse is received 'immediately' in the target-cells. The higher efficacy of the SW-pulse is also supported by the findings of nanotubular intra- and intercellular cell-connections and the mitochondria located inside those tubes $[7,8]$. These nanotubular structures are 'waveguides' also known from the function of SW in DNA and discussed in Meyl [1,2]. In the future further research is necessary to strengthen the hypothesis made here and to apply these data for medical advancements.

\section{References}

1. Meyl K (2012) Cellular Communication, signaling and control from the differen perspectives of Biology, Chemistry and Field Physics. WMSCI Proceedings 2: 113-117.

2. Meyl K (2010),“DNA und Zellfunk“, Indel Verlagsabteilung.

3. Meyl K, Schnabl H (2015) Biological signals transmitted by longitudinal waves influencing the growth of plants, SEEK Digital Library. Int J Environ Eng 2: 23-27.

4. Ebbers JA, Meyl K (2014) Drug effects in yeast mediated by scalar waves. Med Sci 8: 58-62.

5. Meyl K, Schnabl H (2015) Biological signals transmitted by a resonant L-C-oscillator, PIERS draft proceedings, progress in electromagnetic research, prague, Czech Republic pp: 2601-2606.

6. Schnabl H, Meyl K (2018) Longitudinal magnetic waves trigger higher ATP-levels and extend the aging process of plants. Biol Eng Med 3: 1-4.

7. Scholkmann F (2016) Long range physical cell-to-cell signalling via mitochondria inside membrane nanotubes: a hypothesis. Theor Biol Med Model 13: 16. [Crossref]

8. Wang X, Gerdes HH (2012) Long-distance electrical coupling via tunneling nanotubes. Biochim Biophys Acta 1818: 2082-2086. [Crossref]

9. Kuklinski B (2017) Mitochondrien. Aurum Verlag pp: 30-38

10. https://en.wikipedia.org/wiki/Anthocyan

Copyright: (C2019 Schnabl H. This is an open-access article distributed under the terms of the Creative Commons Attribution License, which permits unrestricted use, distribution, and reproduction in any medium, provided the original author and source are credited. 